\title{
Video Article \\ Studying the Hypothalamic Insulin Signal to Peripheral Glucose Intolerance with a Continuous Drug Infusion System into the Mouse Brain
}

\author{
Reni Ajoy ${ }^{1,2}$, Szu-Yi Chou ${ }^{1,2,3}$ \\ ${ }^{1}$ The Ph.D. Program for Neural Regenerative Medicine, College of Medical Science and Technology, Taipei Medical University and National Health Research \\ ${ }^{2}$ Graduate Institute of Neural Regenerative Medicine, College of Medical Science and Technology, Taipei Medical University \\ ${ }^{3}$ TMU research center for Neurotrauma and Neuroregeneration, College of Medical Science and Technology, Taipei Medical University
}

Correspondence to: Szu-Yi Chou at sichou@tmu.edu.tw

URL: https://www.jove.com/video/56410

DOI: doi:10.3791/56410

Keywords: Medicine, Issue 131, Brain drug delivery, micro-osmotic pump, insulin tolerance, glucose metabolism, chemokine (C-C motif) ligand 5 (CCL5), type 2 diabetes mellitus (T2DM)

Date Published: $1 / 4 / 2018$

Citation: Ajoy, R., Chou, S.Y. Studying the Hypothalamic Insulin Signal to Peripheral Glucose Intolerance with a Continuous Drug Infusion System into the Mouse Brain. J. Vis. Exp. (131), e56410, doi:10.3791/56410 (2018).

\section{Abstract}

Insulin regulates systematic metabolism in the hypothalamus and the peripheral insulin response. An inflammatory reaction in peripheral adipose tissues contributes to type 2 diabetes mellitus (T2DM) development and appetite regulation in the hypothalamus. Chemokine CCL5 and C-C chemokine receptor type 5 (CCR5) levels have been suggested to mediate arteriosclerosis and glucose intolerance in type 2 diabetes mellitus (T2DM). In addition, CCL5 plays a neuroendocrine role in the hypothalamus by regulating food intake and body temperature, thus, prompting us to investigate its function in hypothalamic insulin signaling and the regulation of peripheral glucose metabolism.

The micro-osmotic pump brain infusion system is a quick and precise way to manipulate CCL 5 function and study its effect in the brain. It also provides a convenient alternative approach to generating a transgenic knockout animal. In this system, CCL5 signaling was blocked by intracerebroventricular (ICV) infusion of its antagonist, ${ }^{\text {Met }}$ CCL5, using a micro-osmotic pump. The peripheral glucose metabolism and insulin responsiveness was detected by the Oral Glucose Tolerance Test (OGTT) and Insulin Tolerance Test (ITT). Insulin signaling activity was then analyzed by protein blot from tissue samples derived from the animals.

After 7-14 days of ${ }^{\mathrm{Met}}$ CCL5 infusion, the glucose metabolism and insulin responsiveness was impaired in mice, as seen in the results of the OGTT and ITT. The IRS-1 serine302 phosphorylation was increased and the Akt activity was reduced in mice hypothalamic neurons following CCL5 inhibition. Altogether, our data suggest that blocking CCL5 in the mouse brain increases the phosphorylation of IRS-1 S302 and interrupts hypothalamic insulin signaling, leading to a decrease in insulin function in peripheral tissues as well as the impairment of glucose metabolism.

\section{Video Link}

The video component of this article can be found at https://www.jove.com/video/56410/

\section{Introduction}

Insulin affects a wide variety of tissues including the brain. Insulin passes through blood-brain barrier, enters the central nervous system (CNS), and binds with insulin receptor (IR) in the hypothalamus to regulate food intake, sympathetic activity, and peripheral insulin response. Chronic inflammation in peripheral adipose tissues has been proposed to contribute to type 2 diabetes mellitus (T2DM), but how these inflammatory reactions affect insulin signals in the hypothalamus to mediate systemic insulin response and glucose intolerance remains unclear. Some chemokines participate in appetite regulation and body temperature regulation in the hypothalamus ${ }^{1}$ such as tumor necrosis factor-alpha (TNF $\alpha$ ), interleukin (IL)-6, IL-1 $\beta$, monocyte chemoattractant protein-1 (MCP-1), and CCL5 (C-C motif ligand 5). In addition, inflammation in the hypothalamus leads to insulin resistance in $\mathrm{T}_{2} \mathrm{DM}^{2,3}$.

Among these chemokines, the alteration of expression levels of chemokine CCL5 and its receptor, CCR5, in adipose tissues has been associated with arteriosclerosis and glucose intolerance in T2DM in humans as well as animals. CCL5 also has neuroendocrine functions, including the regulation of food intake and body temperature, in the hypothalamus. It is thus important to investigate whether CCL5 participates in insulin signal activation within the hypothalamus or the peripheral tissues.

Insulin signaling is tightly regulated within cells. The binding of insulin to insulin receptors (IR) activates insulin receptor substrate (IRS) proteins, followed by phosphatidylinositol 3-kinase (PI3K) and protein kinase B (PKB/AKT) activation and glucose transporter-4 (GLUT4) membrane translocation ${ }^{4}$. IRS proteins are the key regulators in this signaling pathway: they have multiple tyrosine and serine residues, which can be phosphorylated in response to positive or negative insulin signals ${ }^{5}$. For example, serine 302 phosphorylation on IRS-1 can lead to the physical dissociation of IRS-1 from IR and block insulin signal transduction, leading to insulin resistance ${ }^{6}$. The activity impairment of IRS proteins in the hypothalamus has been shown to induce insulin resistance and glucose intolerance in mice ${ }^{7}$. 
One common way to study the function of a specific gene is the manipulation of the expression of target genes distributed throughout the entire body of the organism. However, this may have several drawbacks: 1) it could generate different feedback regulatory or compensatory effects over time and 2) this method does not help us illustrate the role of the target protein in the specific brain regions. Also, tissue- and cellspecific gene knockout animals take a long time to breed and are costly. Thus, we use a short-term brain infusion osmotic pump system - a relatively quick and convenient way to interfere with the signaling of the target protein in the brain using the antagonist drug to overcome the aforementioned issues. Stereotactic injections used to require intricate surgical skill and extensive investment in instrumentation and time. In this protocol, we provide a simple and safe way to perform stereotactic injection and a quick, less harmful, and instantaneous method to detect the concentration of blood glucose and investigate the role of CCL5 in hypothalamic insulin signaling regulation.

\section{Protocol}

NOTE: All the protocols and methods used in animal subjects have been approved by Institutional Animal Care and Use Committees (IACUC) of the Taipei Medical University (Protocol numbers: LAC-2014-0387)

\section{Preparation of Micro-Osmotic Pump Infusion Systems}

NOTE: Prepare the pump, artificial cerebrospinal fluid (aCSF) buffer, and drug (Met-CCL5/RANTES protein solution (10 $\mathrm{ng} / \mathrm{mL}$ in aCSF)) under sterile conditions using buffers filtered with $0.2 \mu \mathrm{m}$ filters and conduct all the procedures under the culture hood with gloves. The surgery procedures are conducted as follows:

1. Prepare the micro-osmotic pumps one day before surgery: Fill the brain micro-osmotic pump with artificial cerebrospinal fluid (aCSF) with a 1 $\mathrm{mL}$ syringe and blunt-head needle provided along with the kit. Immerse the micro-osmotic pump in aCSF and place it on a shaker and gently shake overnight.

CAUTION: The pump should be filled with aCSF, and air bubbles should be avoided inside the pump (Figure 1A).

2. Before starting surgery, prepare the recombinant Met-CCL5/RANTES protein solution ( $10 \mathrm{ng} / \mathrm{mL}$, diluted in aCSF) to be used in the experiment. Remove aCSF from the pump and fill the pump with the drug solution slowly until excess leaks out.

NOTE: $15 \mathrm{~mL}$ aCSF or Met-CCL5/RANTES solution is sufficient for $5-8$ pumps.

CAUTION: Repeat the procedure to ensure that the pump is completely filled with the drug without bubbles inside.

3. Cut the catheter tubes into the desired length and attach them with the blunt-end brain infusion needle in the brain infusion kit. Fill the infusion kit and tubes with the drug.

4. Finally, assemble and attach the brain infusion kit to the micro-osmotic pump. CAUTION: No bubbles should be formed in the tube or the pump (Figure 1A).

5. Immerse the entire osmotic pump-brain fusion set in aCSF in a sterilized $50 \mathrm{~mL}$ tube to prevent the pump from drying out. The osmotic pumpbrain fusion set is now ready to be used for surgery.

NOTE: The micro-osmotic pump systems can be used for long-term drug infusion. This ensures a safe and convenient mode of drug delivery into the mouse brain.

\section{Intracerebral Ventricular Surgery - implantation of the micro osmotic pump}

CAUTION: Sterilize the surgical environment with $75 \%$ ethanol and ensure that the people involved in the study are wearing sterile gloves and a clean lab coat. Surgical tools/ instruments must be autoclaved and dried before use, and subsequently sterilized with $75 \%$ ethanol in-between mice surgeries.

1. Weigh the mouse and anesthetize it using intra-peritoneal injection (IP) with Ketamine/Xylazine (Ketamine $50 \mathrm{mg} / \mathrm{kg}$, Xylazine $10 \mathrm{mg} / \mathrm{kg}$ ). CAUTION: Mice body weights lower than $24 \mathrm{~g}$ are not recommended for osmotic pump implantation surgery.

2. Mount and fix the mouse head onto the stereotactic apparatus (Figure 1B).

3. Use a pair of surgical scissors and pincers to cut open the outer skin covering the skull. Use iodine to clean the peripheral skull.

4. Separate the outermost layer of skin from the subcutaneous skin with the help of a pair of blunt-head pincers near the neck region for the osmotic pump-brain fusion set implantation (Figure 1C).

5. Mark the infusion point with reference to the brain map (Figure 1D) using the stereotactic apparatus. In this experiment, the needle needs to be implanted in the $3^{\text {rd }}$ ventricle region (Bregma: $0.0 \mathrm{~mm}$ lateral, $1.3 \mathrm{~mm}$ posterior, $5.7 \mathrm{~mm}$ ventral).

6. Drill a hole using a nail drill around the area marked on the skull (Figure 1E).

CAUTION: Be careful not to break the mouse meninges and blood vessels, thus avoiding the disruption of micro-blood vessels in the brain.

7. Place the micro-osmotic pump-brain fusion set containing aCSF (as control) or drug (Met-CCL5/RANTES protein solution) under the skin behind the neck region and insert the brain infusion needle into the drilled hole to infuse the drug into the mouse brain (Figure 1E). The needle will penetrate the meninges and get into the ventricle. Fix the needle in place on the skull using surface desensitizing gel (Figure 1F) and wait 1-2 min until the glue dries. Next, cut off the projecting part on top of the needle (Figure 1G-H).

8. Use a tissue adhesive glue to heal the operation wound on the head. Apply $50 \mu \mathrm{L}$ of the glue on top of the wound, pull both sides of the skin together, and hold on for $30 \mathrm{~s}$ to allow the skin to seal (Figure 1I).

CAUTION: Use $100 \%$ alcohol pad to clean the wound after surgery and $100 \mathrm{ul}$ penicillin with streptomycin to prevent infection. NOTE: Mouse skin will form scar tissue and heal in a few days following the administration of the surgical glue. The main advantage of the glue is the avoidance of surgical stitches which may cause skin irritation or inflammation.

9. Place the mouse in a clean cage kept on a warm plate (heated up to $37^{\circ} \mathrm{C}$ ) and wait until the mouse recovers from the anesthetic effect. CAUTION: It is critical to maintain the mouse's body temperature to enhance the chance of survival after surgery.

10. After a one-week recovery period, the mice will be ready for further experiments, such as the Oral Glucose Tolerance Test (OGTT) and Insulin Tolerance Test (ITT) 


\section{Oral Glucose Tolerance Test (OGTT)}

NOTE: Perform the oral glucose tolerance test 7 days after the infusion of aCSF and ${ }^{\text {Met }}$ CCL5/RANTES $(10 \mathrm{ng} / \mathrm{mL}, 100 \mu \mathrm{L})$. Maintain a $6 \mathrm{~h}$ fast for the mice before OGTT with sufficient water supply. Keep the animals on the same work bench where the experiments will be performed so that they can acclimatize to the environment to reduce stress during the procedure.

1. Glucose solution preparation: Before conducting the experiment, prepare the glucose solution by dissolving $3.75 \mathrm{~g}$ glucose in $15 \mathrm{~mL}$ distilled $\mathrm{H}_{2} \mathrm{O}$.

2. Set up a timetable to record the readings during the experimental procedure (Table 1).

NOTE: It is important to set up a time-table with proper intervals between each blood examination to allow accurate recording during the experiment.

3. Weigh each mouse after fasting and calculate the appropriate amount of glucose to be injected. For example, if the mouse weighs $30 \mathrm{~g}$, the amount of glucose solution to be administered should be $300 \mu \mathrm{L}$.

4. Prepare the following instruments at the work bench:

1. Glucometer (Press the start button to check the battery status, make sure it is functioning before the test.)

2. Glucose chip

3. Insulin syringe $(0.3 \mathrm{~mL}$ Insulin syringe $)$

4. Razor blades

5. Timer

5. Once the bench is set up, measure and record the blood glucose level as follows: Put a clean and new glucose chip into the glucometer and press the start button to zero it.

6. Pick up the mouse by the back of the neck and stroke the tail a few times to ensure sufficient blood flow to the tail region.

7. Using a new razor blade to cut off a small piece of the tail and squeeze out one small drop of blood (about 10-20 $\mu \mathrm{L}$ ) into the glucose chip. The blood should fill the chip to allow accurate measurement. The glucometer will display the glucose level immediately. If the machine shows "error", repeat the procedure with a new glucose chip.

NOTE: The glucose chip requires only one drop of blood. When the blood sample needs to be collected more than once, simply apply pressure by running your fingers along the mouse's tail several times while holding the end of the tail directly on top of the chip to collect blood. It is not necessary to cut the tail end each time while collecting blood samples.

8. Next, feed the mice glucose $(0.25 \mathrm{~g} / \mathrm{mL})$ orally by using the intragastric gavage technique. The amount of glucose to be administered should be calculated by using the formula: 10X body weight (BW) $\mu \mathrm{L}$ glucose solution (for example, if the mouse weighs $30 \mathrm{~g}$, the amount of glucose solution to be administered will be $300 \mu \mathrm{L}$ ). Start the timer immediately after administering oral glucose.

9. Repeat the glucose measurement procedure at $15,30,60,90$, and $120 \mathrm{~min}$.

10. After all the glucose level readings have been recorded, discard the razor blades and glucose chips in a biohazard container. Put the food back into the mice cages and return them to the animal room.

\section{Insulin Tolerance Test (ITT)}

NOTE: The insulin tolerance test and oral glucose tolerance test should be scheduled at least 7 days apart to reduce the fasting effect on animals. For the insulin tolerance test (ITT), human insulin $(0.75 \mathrm{U} / \mathrm{Kg})$ will be administered through IP injection.

1. Preparation of $0.25 \mathrm{U}$ insulin solution: Dilute $100 \mathrm{U}$ human insulin to the ratio of 1:400 in saline solution.

2. Weigh each mouse after fasting, and calculate the injected amount of insulin accordingly: the volume $(\mu \mathrm{L})$ of $0.25 \mathrm{U}$ insulin to be injected IP = $3 \mathrm{X} \mathrm{BW}(0.75 \mathrm{U}$ insulin $/ \mathrm{Kg}$ body weight). For example: for a mouse weighing $28.8 \mathrm{~g}$, inject: $28.8 \times 3=86.4 \mu \mathrm{L}(0.25 \mathrm{U}$ diluted insulin) (Table 2). CAUTION: The same animals might have different body weights after $6 \mathrm{~h}$ fasting on different days. Thus, it is necessary to measure the body weight right before and after fasting and conduct the OGTT and ITT test. Mouse body weight could drop depending on the species, gender, and fasting duration. Higher dosages of insulin could cause insulin shock and would lead to the death of the animal.

3. Set up a table (Table 2) to record the readings during the experimental procedure. Repeat the steps 3.4. to 3.8. for the measurement blood glucose levels.

\section{Representative Results}

Surgical implantations of osmotic infusion pumps containing either aCSF as control or CCL5 antagonist ${ }^{\text {Met }}$ CCL5 (to block CCL5 effects in the brain) were conducted on the mice. At 7 and 14 days after surgery, the peripheral glucose tolerance and insulin responsiveness of mice were analyzed using OGTT (after 7 days) and ITT (after 14 days) as mentioned in the protocol. The oral glucose tolerance test (OGTT) and insulin tolerance test (ITT) of mice were performed after 6 hours fasting. Mice were administered with glucose orally, with the amount based on their respective body weights. The changes in blood glucose levels were recorded, as shown in Figure 3 . The insulin sensitivity test was performed by intraperitoneal (IP) insulin injection into mice and the change of blood glucose level was measured immediately. The changes of blood glucose levels upon insulin stimulation in mice with different infusion drugs were recorded, as shown in Figure 4. The blood glucose level was only slightly reduced after glucose administration (Figure 3B) and insulin injection (Figure 4B) in mice with CCL5 antagonist ( ${ }^{\text {Met }}$ CCL5) infusion as compared to mice with aCSF infusion. These results suggest impairments in insulin function on peripheral glucose metabolism in mice with ${ }^{\mathrm{Met}} \mathrm{CCL} 5$ administered in the brain. 
Next, we analyzed the activation of insulin signal by evaluating the IRS-1 phosphorylation and Akt activation levels in hypothalamic tissues. The serine phosphorylation on 302 of IRS-1 was upregulated in mice treated with antagonist $\left({ }^{\text {Met }}\right.$ CCL5) (Figure 5B-C) when mice were fed normally. In the control group, aCSF was administrated to the mice hypothalamus and the insulin challenge activated the downstream signal molecule Akt (phosphorylated Akt Serine 473) (Figure 5D, F) without increasing IRS-1 serine302 activation (Figure 5D-E) and Akt serine473 phosphorylation. In contrast, the Akt signal was not increased in mice infused with ${ }^{\text {Met }}$ CCL5, but there was an increase in phosphorylation of IRS-1 serine 302 instead. Meanwhile, blocking the CCL5 signal in the mouse brain interrupted insulin activity in the hypothalamus and impaired peripheral insulin function. From our overall findings, such as the results from the ITT, OGTT, and ex vivo insulin challenge, we concluded that CCL5 in the hypothalamus contributes to insulin signal activation and peripheral glucose metabolism upon insulin stimulation.
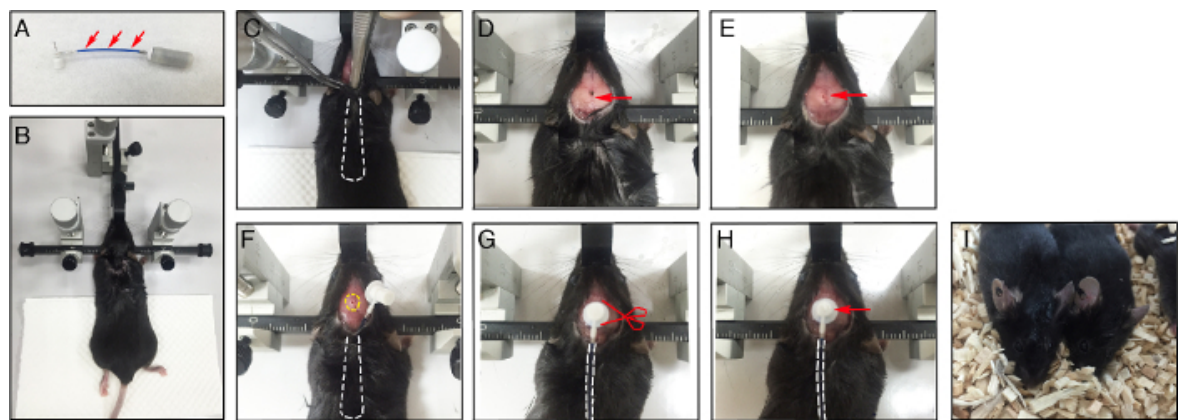

Figure 1. Osmotic pump preparation and implantation surgical procedure in mouse. (A) The brain infusion kit and pump preparation perfused with drug solution. Red arrows indicate the catheter tubes filled with liquid. (B) Fix and mount the mouse head onto the stereotactic apparatus. (C) Separate the outermost layer of the skin from the subcutaneous skin for the implantation of micro-osmotic pump-brain infusion set; dash lines indicate the location of osmotic pump implants. (D) The arrow indicates the infusion side. (E) Drill a hole around the marked area on the skull. (F) Place the osmotic pump-brain infusion set into the back of the mouse and insert the brain infusion needle into the drilled hole (dash circled). (G) Fix the needle onto the skull using tissue-adhesive glue and detach the top of the needle (Scissor pointed in G) as shown in (H). (I) Seal the wound using tissue adhesive glue. Please click here to view a larger version of this figure.
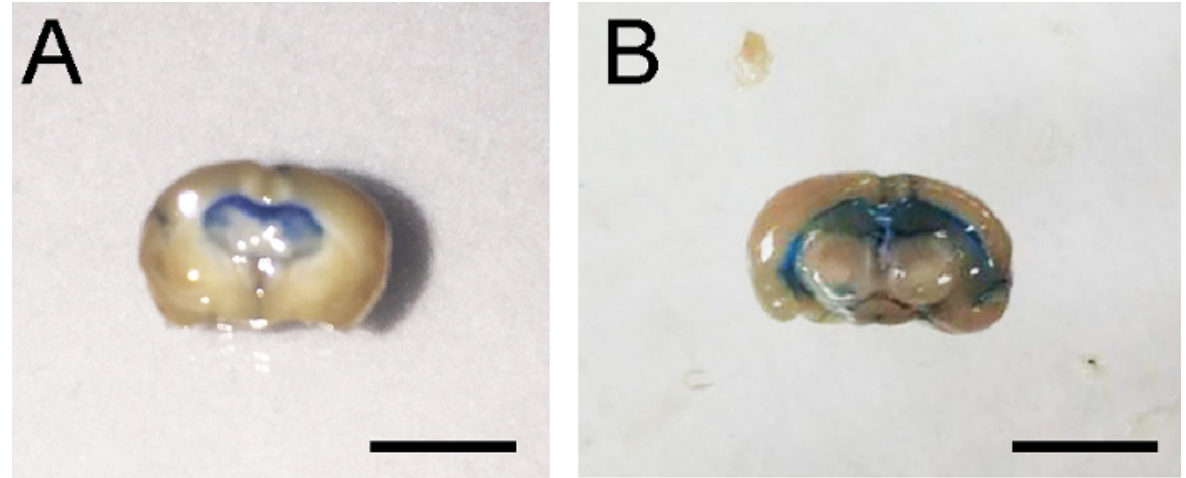

Figure 2. Representative images of the drug diffusion area when the drug is administered in the ventricular region using the osmotic pump. Evan's blue is the representative drug used in the osmotic pump drug infusion illustration into the ventricular region (A) and diffusion into lateral and third ventricles $(\mathbf{B})$. Scale bar $=0.5 \mathrm{~cm}$. Please click here to view a larger version of this figure.

A

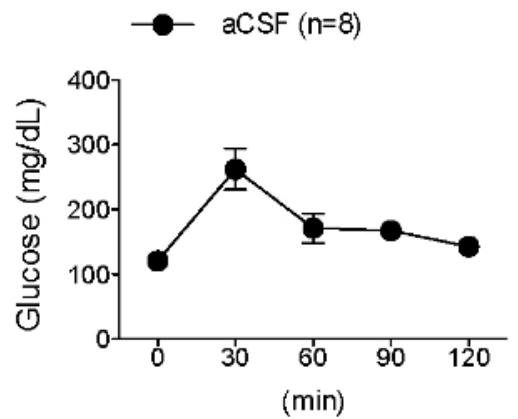

$\mathrm{B}$

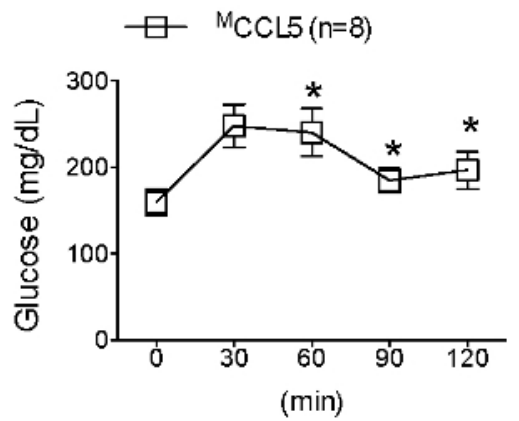

Figure 3. Glucose metabolism of the mice after surgery measured by the oral glucose tolerance test (OGTT). The distribution of blood glucose levels changed following the oral administration of glucose in WT mice infused with aCSF (A) and antagonist, ${ }^{\mathrm{M}} \mathrm{CCL} 5$ (B). Data shown as mean \pm $\mathrm{SE}$. (Figure modified from ${ }^{8}$ ). ${ }^{*} \mathrm{p}<0.05$, by two-way ANOVA. Please click here to view a larger version of this figure. 
A

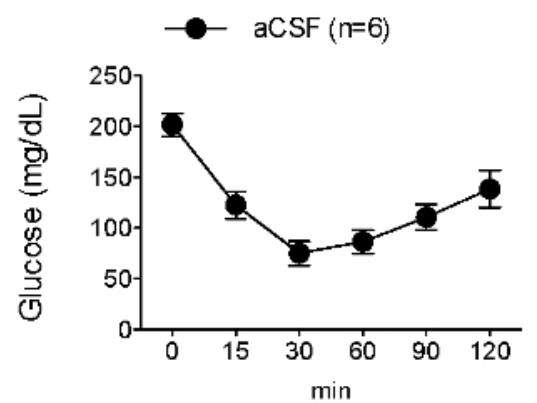

B

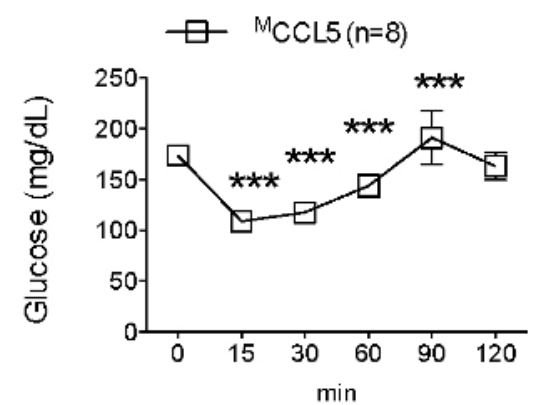

Figure 4. Insulin function in mice blood glucose - the insulin tolerance test (ITT). The distribution of blood glucose levels changed following insulin injection in WT mice infused with aCSF (A), and infused with antagonist, ${ }^{\mathrm{M}} \mathrm{CCL} 5$ (B). Data presented as mean \pm SE (Figure modified from $^{8}$ ). ${ }^{* \star} p<0.001$, by two-way ANOVA. Please click here to view a larger version of this figure.

A

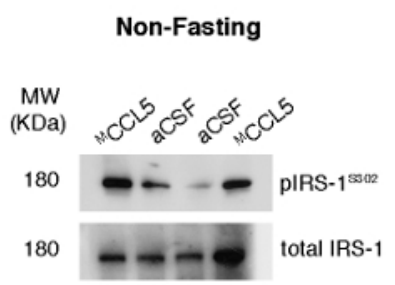

C

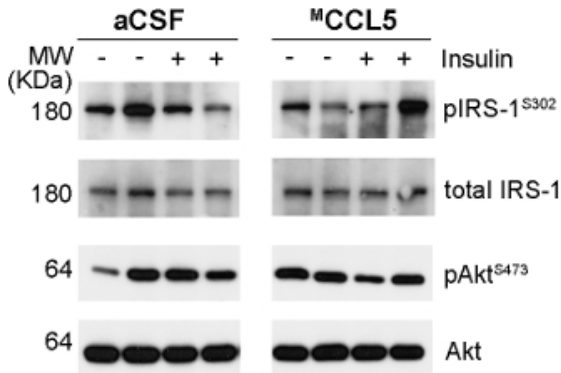

B

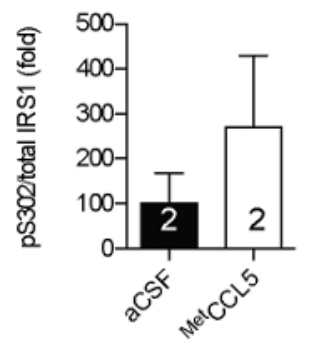

D

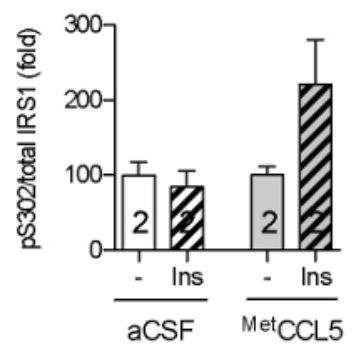

$E$

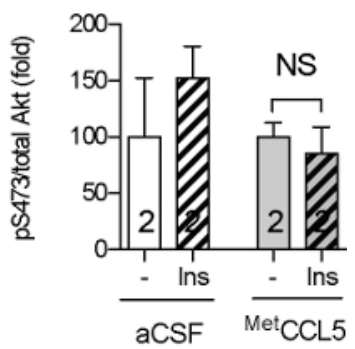

Figure 5. Insulin signal activity in mice after surgery. (A) Western blotting of the inhibitory Serine 302 phosphorylation form of IRS-1 (insulin response substrate-1, pIRS $1{ }^{\mathrm{S} 302}$ ) in mice hypothalamus tissues treated with aCSF or CCL5 antagonist, ${ }^{\text {Met }}$ CCL5 ( ${ }^{\mathrm{M}}$ CCL5) infusion pump. (B) Related levels of phosphor-IRS-1 ${ }^{\mathrm{S} 302}$ after infusion in mice hypothalamus with normal feeding. (C) Western blotting of the S302 phosphorylation of IRS-1 and Akt activation (phosphor-Akt S473, pAkt ${ }^{\text {S473 }}$ ) with or without insulin stimulation in hypothalamic tissue after aCSF or ${ }^{\text {MET }}$ CCL5 infusion. (D-E) Relative levels of pIRS-1 ${ }^{\mathrm{S} 302}, \mathrm{pS}^{\mathrm{T}} \mathrm{K}^{\mathrm{T} 21}$, and $\mathrm{pAkt} \mathrm{s}^{\mathrm{S43}}$. ("2" in each bar graph stands for: $\mathrm{n=2}$ for all quantifications). (The blank bars in 5D-E, left: without insulin; the strips bars in 5D-E, right: with insulin). Please click here to view a larger version of this figure.

\begin{tabular}{|c|c|c|c|c|c|c|c|c|c|}
\hline$\#$ & Mouse ID & Body & Glucose & Start & 0 & 15 & 30 & 60 & 90 \\
\hline & & weight & $\mu L=10 x B W$ & time & mins & mins & mins & mins & mins \\
\hline 1 & 501 & $25.8 \mathrm{~g}$ & 258 & $9: 00$ & $9: 00$ & 9:00 & $9: 15$ & $9: 30$ & $10: 00$ \\
\hline 2 & 502 & $25.3 g$ & 253 & 9:07 & $9: 07$ & 9:07 & $9: 22$ & $9: 37$ & 10:07 \\
\hline
\end{tabular}

Table 1. Timetable for Oral Glucose Tolerance Test (OGTT) recording

\begin{tabular}{|l|l|l|l|l|l|l|l|l|l|}
\hline$\#$ & Mouse ID & Body & $\begin{array}{l}\text { Insulin } \\
\mathbf{0 . 2 5 I U}\end{array}$ & Start & $\mathbf{0}$ & $\mathbf{1 5}$ & $\mathbf{3 0}$ & $\mathbf{6 0}$ & $\mathbf{9 0}$ \\
\hline & & weight & $\mathbf{\mu L}=\mathbf{3 x B W}$ & time & mins & mins & mins & mins & mins \\
\hline 1 & 501 & $28.8 \mathrm{~g}$ & 86.4 & $9: 00$ & $9: 00$ & $9: 15$ & $9: 30$ & $10: 00$ & $10: 30$ \\
\hline 2 & 502 & $25.3 \mathrm{~g}$ & 75.9 & $9: 07$ & $9: 07$ & $9: 22$ & $9: 37$ & $10: 07$ & $10: 37$ \\
\hline
\end{tabular}

Table 2. Timetable for Insulin Tolerance Test (ITT) recording 
Discussion

The mechanism of chronic inflammation and related chemokines such as CCL5 and its receptor - CCR5 in the development of type-2 diabetes remains unclear. Chronic inflammation causes macrophage infiltration into adipose tissues and affects the regulation of adipokines; in the meantime, it also attracts $\beta$-cells and impairs insulin secretion from the islets of Langerhans in response to blood glucose. Hypothalamus in the brain plays an important role as a control center in coordinating insulin and adipokine signals from the systemic peripheral tissues in regulating appetite, peripheral blood glucose metabolism, and insulin response. Many studies also indicate that hypothalamic inflammation leads to defective regulation of energy homeostasis as well as defective pancreatic islet and liver function ${ }^{2,3,9,10}$. CCL5 in the brain contributes to food intake and body temperature regulation in the hypothalamus ${ }^{11,12}$; however, the correlation of CCL 5 to hypothalamic and systemic insulin signaling is unclear. A CCL5 whole body knockout mouse $\left(\mathrm{CCL} 5^{-/-}\right)$has been generated to address this question, which shows an insulin resistance phenotype with higher insulin levels and high blood glucose levels in blood ${ }^{8}$. However, it requires a long time to develop the T2DM phenotype and it is difficult to investigate the role and mechanism of CCL5 in hypothalamic insulin signal due to possible long-term compensatory effects. Therefore, a direct manipulation of CCL5 signaling in hypothalamic neurons is the best approach. There are, however, multiple types of neurons in the hypothalamic region and it is quite expensive and time-consuming to generate cell specific knockout mice. Utilizing an ICV infusion system can thus save time and provide a more specific approach to manipulate CCL5 function directly in the brain, bypassing possible peripheral inflammatory reactions.

Studies utilizing osmotic pumps have already been published previously, providing great examples and demonstrations of techniques involved in the implantation of osmotic pumps in rodents ${ }^{13}$. However, we faced a few challenges while following these protocols in our study. First, some of the equipment used in the protocol is quite expensive, including 1) the electric system to reach the location, drawing and inserting the needle into mouse brain, 2) the thermo system for maintaining mouse body temperature and 3) the oxygen-isoflurane supply system for administering anesthesia to mice. Second, the techniques described in other articles were difficult to replicate because we were only able to use animals within a small range of body weights and at certain ages for our study. We are aware that larger mice are more suitable for surgery and implantation. However, in our study, we had to use smaller and younger mice to avoid the overweight and aging effects on insulin and blood glucose regulation: only male mice with body weight $25 \pm 2 \mathrm{~g}$ and age around 2 months old were chosen in the study. Thus, it is difficult to perform surgery and suture the wound on the mouse head. Third, the inflammatory response has to be minimized after surgery since an inflammatory cytokine is the target in this study. Mice and rats can remove suture and open wounds easily after surgery, which will result in inflammation and increase chemokine reactions. Therefore, a strategy to reach the location and draw and insert the needle into mouse brain that avoids secondary infection is necessary. Therefore, we modified the previously described protocols to make this technique cost effective, easier, and less harmful to the animals, as described in the following paragraph.

Firstly, we used a nail drill to manually drill a hole around the target area marked on the skull, as described in step 2.6. This method is cost effective and allows us to monitor the entire procedure so as to avoid damaging the mouse meninges and blood vessels. Blood glucose regulation is impaired after acute stroke, such as a hemorrhage in the brain. Acute hyperglycemia and diabetes-like syndromes were also observed after stroke in clinical settings ${ }^{14,15}$. Similarly, we also found impaired glucose level and insulin response in mice with hemorrhage and pus in the brain. We are aware that better control of manual-based surgery is necessary to ensure consistency of the results. Secondly, we took advantage of a newly developed medical biomaterial commonly used in clinics, tissue adhesive glue (Step 2.8), to seal the skin on the mouse head following surgery, hence, avoiding stitches and accelerating the rate of healing. This makes surgical procedures easier to perform and reduces the chance of secondary inflammation. Thirdly, the time required to perform the entire surgical procedure is comparatively shorter, which increases the chance of survival for the mice and lowers the dosage of anesthetic drug being injected intraperitoneally. We observed a high survival rate $(95 \%)$ and obtained relatively accurate results by following this modified protocol.

The limitation of this technique is the relatively short time frame of drug delivery. Although an osmotic pump can be placed into the mouse body alternatively without re-opening the brain, our study only focuses on the inflammatory chemokine effect on the brain to regulate the peripheral systemic insulin signaling. Additional surgery in peripheral tissues could possibly induce an inflammatory reaction in peripheral tissues, which would then increase inflammatory chemokine expression and affect the results. Secondly, the half-life of the drug also limits the duration of the study. Recombinant proteins such as chemokine usually have a shorter half-life, which loses its activity over time, though it also allows us to study the effect of blocking CCL5 signaling in the brain over the short term. Our previous studies have also described a genetic modification approach for generating a CCL5 knockout mouse, which provides a model with long-term effects ${ }^{8}$.

There are some new techniques and alternative methods to deliver drugs into the brain. Nanotechnology is a powerful technique, which can be used to deliver drugs into the central nervous system. However, many drugs are thermosensitive and can be destroyed when trying to package them into nanoparticles ${ }^{16}$. In addition, nanoparticles can pass through BBB and be uptaken by cells that are suitable for siRNA or most common drugs, but it is not an ideal method for ligand-receptor binding. CCL5 requires binding to its receptor, CCR5, in the hypothalamus ARC neurons to take effect ${ }^{8}$, and the delivery of CCL5 antagonist ${ }^{\text {Met }}$ CCL5 into neurons through nanoparticles might cause a loss of the ability to bind and block CCR5 on the cell surface.

The blood glucose level was significantly higher in mice administered with the CCL5-antagonist ${ }^{\text {Met }}$ CCL5 as compared to the controls (mice administered with aCSF) in the oral glucose tolerance test. Additional insulin administration (insulin tolerance test) was also unable to lower the blood glucose level in ${ }^{M e t}$ CCL5 receiving mice (Figure 4B), which suggests that both endogenous and external insulin cannot reduce blood glucose levels when blocking hypothalamic CCL5 signaling. Mice became insulin resistant without CCL5 activity in the hypothalamus. Increased serine302 phosphorylation of IRS-1 was found in the mice receiving Met-CCL5 compared to control mice receiving aCSF (Figure 5A-B). Serine 302 phosphorylation of IRS-1 has been shown to induce a physical dissociation of IRS-1 from the insulin receptor, which is a major cause of insulin resistance ${ }^{6}$; insulin is unable to activate downstream signals such as the PI3K-Akt pathway. An ex vivo insulin stimulation study confirmed the insulin downstream signaling molecule Akt ( $p$-AktS473) was not activated by insulin in mouse hypothalamic tissue infused with Met-CCL5 and, instead, the serine 302 phosphorylation increased. Altogether, both physiological data (OGTT and ITT) and molecular study demonstrate that hypothalamic CCL5 signaling mediates the hypothalamic insulin signal regulation, which contributes to systematic insulin resistance and glucose metabolism. 
The role and mechanism of CCL5 and CCR5 in obesity-associated diabetes remains unclear. Kitade et al. reported that CCR5 deficiency protected mice from obesity-induced inflammation, macrophage recruitment, and insulin resistance ${ }^{17}$. However, other studies by Kennedy et al. found opposite results indicating that CCR5 deficiency impairs systemic glucose tolerance as well as adipocyte and muscle insulin signaling ${ }^{18}$. Both studies applied a high-fat diet to induce obesity, which leads to whole body chronic inflammation and compensatory response. These studies did not provide clean and clear mechanisms of CCL5 and CCR5 in insulin signaling regulation. On the other hand, the osmotic pump technique allows a brain specific infusion and avoids compensatory response with its time-limited delivery.

In conclusion, although the osmotic pump with the brain infusion system seems to be an "old-fashioned" technique, it does provide a cheaper, easier, and less harmful method of drug delivery and helps investigate the function of ligand-receptor signaling in the brain.

\section{Disclosures}

The authors have nothing to disclose.

\section{Acknowledgements}

We are grateful the supported from the Ministry of Science and Technology, Taiwan - MOST105-2628-B-038-005-MY3(1-3) and Health and welfare surcharge of tobacco products - MOHW106-TDU-B-212-144001 to S-Y C.

\section{References}

1. Plata-Salaman, C.R., \& Borkoski, J.P. Chemokines/intercrines and central regulation of feeding. Am J Physiol. 266, R1711-1715 (1994).

2. Milanski, M., et al. Inhibition of hypothalamic inflammation reverses diet-induced insulin resistance in the liver. Diabetes. 61, 1455-1462 (2012).

3. Wang, X., et al. Increased hypothalamic inflammation associated with the susceptibility to obesity in rats exposed to high-fat diet. Experimental diabetes research. 2012, 847246 (2012).

4. Benomar, Y., et al. Insulin and leptin induce Glut4 plasma membrane translocation and glucose uptake in a human neuronal cell line by a phosphatidylinositol 3-kinase- dependent mechanism. Endocrinology. 147, 2550-2556 (2006).

5. Gual, P., Le Marchand-Brustel, Y., \& Tanti, J.F. Positive and negative regulation of insulin signaling through IRS-1 phosphorylation. Biochimie. 87, 99-109 (2005).

6. Werner, E.D., Lee, J., Hansen, L., Yuan, M., \& Shoelson, S.E. Insulin resistance due to phosphorylation of insulin receptor substrate-1 at serine 302. The Journal of biological chemistry. 279, 35298-35305 (2004).

7. Boura-Halfon, S., \& Zick, Y. Phosphorylation of IRS proteins, insulin action, and insulin resistance. American journal of physiology. Endocrinology and metabolism. 296, E581-591 (2009).

8. Chou, S.Y., et al. CCL5/RANTES contributes to hypothalamic insulin signaling for systemic insulin responsiveness through CCR5. Sci Rep. 6 , 37659 (2016).

9. Calegari, V.C., et al. Inflammation of the hypothalamus leads to defective pancreatic islet function. J Biol Chem. 286, 12870-12880 (2011)

10. Mighiu, P.I., Filippi, B.M., \& Lam, T.K. Linking inflammation to the brain-liver axis. Diabetes. 61, 1350-1352 (2012).

11. Tavares, E., \& Minano, F.J. RANTES: a new prostaglandin dependent endogenous pyrogen in the rat. Neuropharmacology. 39, 2505-2513 (2000).

12. Appay, V., \& Rowland-Jones, S.L. RANTES: a versatile and controversial chemokine. Trends in immunology. 22, 83-87 (2001).

13. DeVos, S.L., \& Miller, T.M. Direct intraventricular delivery of drugs to the rodent central nervous system. $J$ Vis Exp., e50326 (2013).

14. Wang, N., et al. Admission blood glucose and in-hospital clinical outcome among patients with acute stroke in Inner Mongolia, China. Clin Invest Med. 32, E151-157 (2009).

15. Olsen, T.S. Blood glucose in acute stroke. Expert Rev Neurother. 9, 409-419 (2009).

16. De Jong, W.H., \& Borm, P.J. Drug delivery and nanoparticles:applications and hazards. Int J Nanomedicine. 3, 133-149 (2008).

17. Kitade, H., et al. CCR5 plays a critical role in obesity-induced adipose tissue inflammation and insulin resistance by regulating both macrophage recruitment and M1/M2 status. Diabetes. 61, 1680-1690 (2012).

18. Kennedy, A., et al. Loss of CCR5 results in glucose intolerance in diet-induced obese mice. Am J Physiol Endocrinol Metab. 305, E897-906 (2013). 